\title{
Enabling the next generation of $\mathrm{cm}$-wavelength studies of high-redshift molecular gas with the SKA
}

\section{Jeff Wagg ${ }^{* 1}$, Elisabete Da Cunha ${ }^{2}$, Chris Carilli ${ }^{3,4}$, Fabian Walter ${ }^{2}$, Manuel Aravena ${ }^{5}$, lan Heywood ${ }^{6,7}$, Jacqueline Hodge ${ }^{8}$, Eric Murphy ${ }^{9}$, Dominik Riechers ${ }^{10}$, Mark} Sargent ${ }^{11}$, and Ran Wang ${ }^{12}$

${ }^{1}$ SKA Organisation, Lower Withington, UK; ${ }^{2}$ MPIA, Heidelberg, Germany; ${ }^{3}$ NRAO, Socorro, USA; ${ }^{4}$ Cavendish Astrophysics Group, Cambridge, UK; ${ }^{5}$ U. Diego Portales, Santiago, Chile;

${ }^{6}$ CASS, Sydney, Australia; ${ }^{7}$ RATT, Dept. of Physics and Electronics, Rhodes University, Grahamstown, South Africa; ${ }^{8}$ NRAO, Charlottesville, USA; ${ }^{9} I P A C$, Caltech, Pasadena, USA; ${ }^{10}$ Cornell University, Ithaca, USA; ${ }^{11}$ Astronomy Centre, Department of Physics and Astronomy, University of Sussex, Brighton, UK; ${ }^{12}$ KIAA, Peking, Beijing, China

E-mail: j.waggeskatelescope.org

\begin{abstract}
The Square Kilometre Array will be a revolutionary instrument for the study of gas in the distant Universe. SKA1 will be among the first facilities with sufficient sensitivity to detect and image atomic $21 \mathrm{~cm} \mathrm{HI}$ in individual galaxies at significant cosmological distances, complementing ongoing ALMA imaging of redshifted high- $J$ CO line emission and far-infrared interstellar medium lines such as [CII] $157.7 \mu \mathrm{m}$. At frequencies below $\sim 50 \mathrm{GHz}$, observations of redshifted emission from low- $J$ transitions of $\mathrm{CO}, \mathrm{HCN}, \mathrm{HCO}^{+}$, $\mathrm{HNC}, \mathrm{H}_{2} \mathrm{O}$ and $\mathrm{CS}$ provide insight into the kinematics and mass budget of the cold, dense star-forming gas in galaxies. In advance of ALMA band 1 deployment ( 35 to $52 \mathrm{GHz}$ ), the most sensitive facility for high-redshift studies of molecular gas operating below $50 \mathrm{GHz}$ is the Karl G. Jansky Very Large Array (VLA). Here, we present an overview of the role that the SKA could play in molecular emission line studies during SKA1 and SKA2, with an emphasis on studies of the dense gas tracers directly probing regions of active star-formation.
\end{abstract}

Advancing Astrophysics with the Square Kilometre Array

June 8-13, 2014

Giardini Naxos, Italy

\footnotetext{
* Speaker.
} 


\section{Gas in galaxies: 2020 and beyond}

Studies of the dense star-forming gas in high-redshift galaxies have been transformed in recent years owing to the increased sensitivity and bandwidth of new submm through cm-wavelenth facilities like the Karl G. Jansky Very Large Array (VLA), the Plateau de Bure Interferometer (PdBI) and the Atacama Large Millimeter/submm Array (ALMA). A few highlights include observations of molecular gas through ${ }^{12} \mathrm{CO}$ (hereafter $\mathrm{CO}$ ) line emission in main sequence star-forming galaxies at $z \sim 1.5-2.0$ (Daddi et al. 2008, 2010; Dannerbauer et al. 2009; Tacconi et al. 2010, 2013; Aravena et al. 2010, 2014), the detection and imaging of CO and [CII] line emission in quasar host galaxies during the epoch of reionization (Walter et al. 2003, 2004; Carilli et al. 2007; Wang et al. 2013; Willott et al. 2013), and the first blank-field CO emission line surveys for gas-rich galaxies over significant cosmological volumes (Walter et al. 2014; Decarli et al. 2014). These new surveys greatly expand our knowledge of the gas content of galaxies in the distant Universe, which had been based primarily on detections of CO in far-infrared luminous AGN host galaxies and submm/mm-selected star-forming galaxies (see Carilli \& Walter 2013 for a recent review). The emerging picture is that, with the exception of atomic HI, the cool gas content of main sequence star-forming galaxies is now accessible with current facilities across the peak in cosmic star-formation. Here, we summarize the molecular gas studies of high-redshift galaxies that could be enabled by the SKA.

In 2020 and beyond, the SKA will operate in parallel with major new facilities covering much of the electromagnetic spectrum, collaboratively conducting sensitive studies of gas and starformation in galaxies well into the epoch of reionization (EoR) at $z>6$. These facilities include optical telescopes like the European Extremely Large Telescope (E-ELT), the Thirty Meter Telescope (TMT), and the Large Synoptic Survey Telescope (LSST), and infrared facilities such as the James Webb Space Telescope (JWST) and Euclid. At long submm/mm-wavelengths, ALMA, NOEMA, the Cerro Chajnantor Atacama Telescope (CCAT) and the Large Millimeter Telescope (LMT) will shed light on Galactic and extragalactic regions of the Universe obscured by molecular gas and dust. The long wavelength complement to these facilities will be the SKA.

One of the main science drivers for the SKA has always been the study of $21 \mathrm{~cm} \mathrm{HI}$ line emission in normal galaxies out to redshifts $z \gtrsim 1$. Predictions and science drivers for future SKA extragalactic HI surveys are presented by other authors in this volume (e.g. Morganti et al. 2015; Obreschkow et al. 2015; Santos et al. 2015; Staveley-Smith et al. 2015). Similarly, the SKA will provide the unique ability to survey thermal and non-thermal (synchrotron) radio emission at low radio frequencies covering 10s to 1000 s of square degrees in order to probe the evolution of obscured star-formation out to redshifts approaching the end of reionization at $z \sim 6$ (see Prandoni \& Seymour 2015, Jarvis et al. 2015, and Murphy et al. 2015 in this volume). Over fields much smaller than one square degree, this obscured star-formation will be probed to very sensitive depths by ALMA, which has already been successful in imaging far-infrared continuum emission in starforming galaxies during the epoch of reionization (e.g. Wang et al. 2013). For galaxy evolution studies, the wide field imaging capabilities of the SKA will be complemented at optical/infrared wavelengths by LSST and Euclid. Finally, ALMA is sensitive to the high- $J \mathrm{CO}, \mathrm{HCN}, \mathrm{HCO}^{+}$ and CS line emission in the distant Universe at frequencies $v>86 \mathrm{GHz}(v>35 \mathrm{GHz}$ once band 1 is deployed), leaving open the need for a sensitive facility able to conduct surveys of the low- 
$J$ transitions of these molecules that provide an anchor for the spectral line energy distribution. Without this anchor, there will be degeneracies in the models fit to derive gas properties such as density and temperature. Measurements of only the high- $J$ CO emission lines may lead to a bias in molecular gas mass estimates as their intensities will depend on the excitation conditions of the gas.

\section{Molecular CO line emission with the SKA}

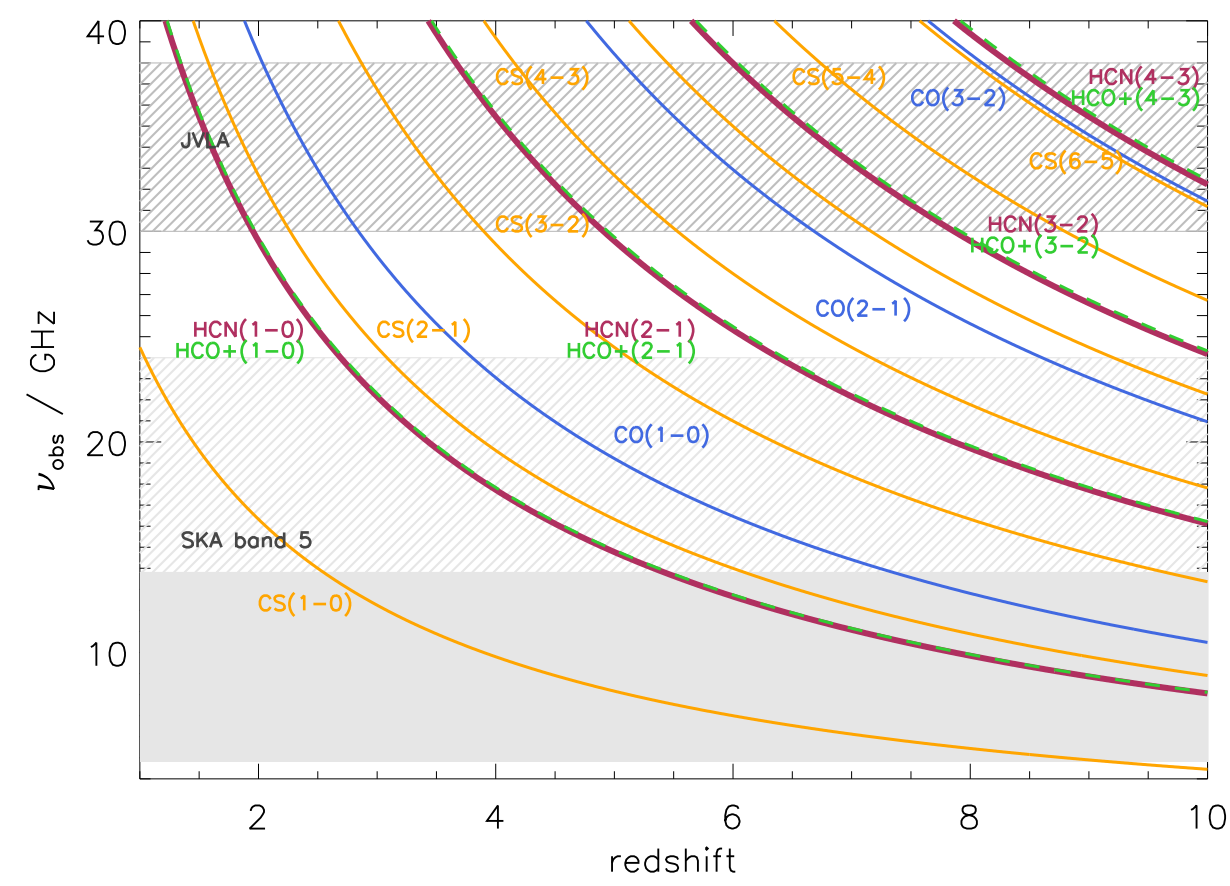

Figure 1: Redshifted line frequencies for multiple low- $J$ transitions of $\mathrm{CO}, \mathrm{CS}, \mathrm{HCN}$, and $\mathrm{HCO}^{+}$. The grey region shows the currently defined frequency range of band 5 on SKA1-MID, and the proposed extension to $24 \mathrm{GHz}$ for SKA2 is shown as a hatched region. At higher frequencies we show the frequency coverage provided by an $8 \mathrm{GHz}$ bandwidth VLA survey with the Ka-band receivers (26.5 to $40 \mathrm{GHz}$ ). ALMA band 1 is expected to cover 35 to $52 \mathrm{GHz}$ (Di Francesco et al. 2013).

Among the outstanding challenges for galaxy formation studies is to quantify the kinematic properties and to measure the gas content of star-forming galaxies over the history of the Universe. Aided by the typical increase in line flux density with increasing rotational transition in star-forming molecular gas, interferometric observations of $\mathrm{CO}$ line emission provide a means of estimating dynamical masses through the linewidths and source sizes in galaxies out to the very early Universe (e.g. Walter et al. 2004; Riechers et al. 2013). At cm-wavelengths, one is sensitive to the low- $J$ transitions of this line, the luminosity of which has traditionally been used as a means of estimating the total cold molecular $\left(=\mathrm{H}_{2}\right)$ mass that is available to fuel star-formation by adopting a CO-to- $\mathrm{H}_{2}$ conversion factor, $\alpha_{C O}$ (e.g Downes \& Solomon 1998; Bolatto et al. 2013). As such, observations of high-redshift molecular CO line emission have naturally become one of 
the main science drivers for current and future cm-wavelength interferometers operating above $\sim 20 \mathrm{GHz}$. Figure 1 shows the redshifted frequencies of the low- $J \mathrm{CO}$ lines, along with those of dense gas tracing molecular line species.

Here, we consider the likelihood that $\mathrm{CO} J=1-0$ line emission will be either a viable tracer of galaxy dynamics or a tool for estimating cold molecular gas masses with the current plans for SKA1-MID and SKA2. The possibility of CO intensity mapping with the SKA is discussed in another chapter (Chang et al.). Five bands are currently defined for SKA1-MID, although only three are expected to be deployed in the first phase. Band 5 would extend up to $13.8 \mathrm{GHz}$, corresponding to $\mathrm{CO} J=1-0$ line emission redshifted to $z=7.35$. The most luminous metal-enriched quasar host galaxy at $z=7.1$ is undetected in CO $J=6-5$ line emission with the PdBI (Venemans et al. 2012), which should benefit from a factor of $\sim 36 \times$ increase in flux density with respect to the CO $J=1-0$ line emission owing to the $v^{2}$ dependance of flux density on rest frequency. Molecular line emission from transitions where the excitation temperature is similar to the CMB background temperature $\left(\mathrm{T}_{\mathrm{CMB}}(\mathrm{z})=2.7(1+\mathrm{z})\right.$ ) should not be detectable (e.g. Papadopoulos et al. 2000; Obreschkow et al. 2009; Da Cunha et al. 2013b). In the case of a $z=7.5$ star-forming galaxy (similar to a submm galaxy or a far-infrared luminous quasar host galaxy) with molecular gas at an average kinetic temperature of $\mathrm{T}_{\mathrm{k}}=40 \mathrm{~K}$ (at $z=0$ ), the CO $J=1-0$ line is predicted to exhibit $\sim 40 \%$ of the flux density it would have in the absence of the CMB. In the case of more quiescent starformation where the kinetic temperature of the gas would be $T_{k}=18 \mathrm{~K}$, the integrated CO J=1-0 line flux density of that galaxy at $z=7.5$ would be down to $5 \%$ of its $z=0$ value. Such effects likely contributed to the (sensitive) Green Bank Telescope (GBT) non-detections of CO $J=1-0$ in two star-forming Ly $\alpha$ emitters (LAEs) at $z>6.5$ by Wagg, Kanekar \& Carilli (2009), including the gravitationally lensed LAE HCM6A, which may have a star-formation rate $\gtrsim 100 \mathrm{M}_{\odot} \mathrm{yr}^{-1}$ (Chary et al. 2005). During these early cosmic times, corresponding to the epoch of reionization (EoR), measuring the kinematic properties (dynamical mass estimates) of quasar host galaxies, or redshifts for the lower luminosity galaxy population, would best be achieved through observations of the redshifted [CII] line emission at mm-wavelengths (e.g. Maiolino et al. 2005; Walter et al. 2009; Wagg et al. 2012a; Carilli et al. 2013; Wang et al. 2013; Riechers et al. 2014).

Adding to the inherent difficulty in detecting the low- $J$ CO lines during the EoR, the average metallicity of galaxies is expected to be lower, and recent observational and theoretical work suggests that $\alpha_{C O}$ may be higher in low metallicity gas clouds (e.g. Genzel et al. 2012; Leroy et al. 2009; Narayanan et al. 2012). Larger $\alpha_{C O}$ naturally leads to a lower CO line flux density per unit mass of $\mathrm{H}_{2}$. The $z>6$ population of star-forming $\mathrm{Ly} \alpha$ emitters have typical star-formation rates of 5-10 $M_{\odot} \mathrm{yr}^{-1}$. Their metallicities cannot be measured with current facilities (except possibly with ALMA, which is sensitive to redshifted FIR lines like [CII] and [NII]), although these are likely to have sub-solar metallicties given the timescales needed for metal enrichment (the Universe is $\sim 710 \mathrm{Myr}$ old at $z=7.5$ ). Wagg et al. (2012b) obtained a sensitive GBT upper-limit on CO $J=2-1$ line emission in the $\mathrm{Ly} \alpha$ blob (LAB) 'Himiko' at $z=6.6$. It is likely that the low expected metallicities of typical $z>6$ star-forming galaxy populations like the LABs and LAEs will mean that SKA1-MID band 5 surveys of CO $J=1-0$ line emission will only be sensitive to more massive and rare starburst galaxies (see Murphy et al. in this edition). The only high-redshift galaxy currently detected in molecular $\mathrm{CO}$ line emission at frequencies below $20 \mathrm{GHz}$ is a gravitationally lensed star-forming submm galaxy at $z=6.34$ (Riechers et al. 2013). The surface density of similarly 
luminous quasar host galaxies detected in CO line emission at $z>6$ is $\sim 1$ per 500 square degrees.

During SKA2, the situation would be improved by the expected order of magnitude increase in collecting area and extended frequency coverage up to $24 \mathrm{GHz}$ (or $30 \mathrm{GHz}$, as proposed by Murphy et al. (2015), who also present the science case for $v>10 \mathrm{GHz}$ extragalactic continuum studies). Such studies would be sensitive to CO $J=1-0$ line emission at $z>3.8(z>2.8$ for $v<30 \mathrm{GHz})$, covering the epoch when massive $z \sim 2$ galaxies should have formed their stars, likely in obscured bursts of star-formation. For the case of cold, quiescent gas at a kinetic temperature of $18 \mathrm{~K}$ in a $z>4$ galaxy, the prediction is that the measured flux density should be less than $25 \%$ of what would be measured in the absence of the CMB (Da Cunha et al. 2013b). For warm star-forming gas at $\mathrm{T}_{\mathrm{k}}=40 \mathrm{~K}$, one would measure $\sim 65 \%$ of the $z=0$ intensity at a redshift of $z=4$. We expect that SKA2-MID should provide a powerful means of quantifying the cosmic evolution of the molecular $\mathrm{H}_{2}$ gas density over the epoch of massive galaxy formation.

\section{Dense star-forming gas}

Dense star-forming gas tracers such as $\mathrm{HCN}$ and CS hold the promise for future SKA studies of the high-redshift interstellar medium (ISM), as their emission lines directly probe sites of active star-formation with densities in excess of $10^{4} \mathrm{~cm}^{-3}$. Although they are typically an order of magnitude fainter than the $\mathrm{CO}$ lines, the luminosity in the $88.6 \mathrm{GHz} \mathrm{HCN} J=1-0$ line correlates linearly with infrared luminosity in star-forming gas over nearly eight orders of magnitude in luminosity (Gao \& Solomon 2004; Wu et al. 2005). As such, emission from lines like HCN $J=1-0$ should be a good proxy for the total mass in dense molecular gas directly involved in ongoing star-formation activity. The HCN $J=1-0$ line traces gas at kinetic temperatures of 40 to $50 \mathrm{~K}$ in luminous infrared star-forming galaxies, much higher than the CMB temperature at redshifts below $z \sim 6$. Current cm-wavelength facilites lack the sensivity to detect HCN $J=1-0$ line emission at $v \lesssim 24 \mathrm{GHz}$ in even the most luminous, known star-forming galaxies and quasar host galaxies. Previous attempts to detect low- $J$ HCN line emission in $z>2$ galaxies have only been successful for a handful of lensed objects (Solomon et al. 2003; Vanden Bout et al. 2005; Carilli et al. 2005; Gao et al. 2007; Riechers et al. 2007), while high- $J$ HCN line emission remains a promising tool for ALMA studies (Wagg et al. 2005; Weiss et al. 2007; Danielson et al. 2011). The $\mathrm{HCO}^{+}$line may also hold promise as a dense gas tracer in high-redshift galaxies (e.g. Riechers et al. 2006; Garcia-Burillo et al. 2006), although the possible decrease in abundance of $\mathrm{HCO}^{+}$in regions of high electron density has brought into question its effectiveness as a proxy for dense molecular gas mass in starburst galaxies (e.g Papadopoulos et al. 2007).

In the case of CS, the $J=1-0$ transition occurs at a rest frequency of $49 \mathrm{GHz}$, close to the atmospheric $\mathrm{O}_{2}$ line, which limits the sensitivity for surveys of CS line emission in the local Universe. CS is a more common tracer of dense star-forming cores in our Galaxy (e.g. Evans 1999), however it has not been widely studied in the high-redshift Universe given the sensitivity of current cm-wavelength instruments.

If band 5 were deployed on SKA1-MID, it would be sensitive to $\mathrm{HCN} J=1-0$ and $\mathrm{HCO}^{+} J=1$ 0 line emission at $z>5.4$ and CS $J=1-0$ line emission at $z>2.6$. However, a more significant advance will be made with the increased sensitivity and extended frequency coverage of SKA2, which should be an excellent facility for the study of low- $J$ transitions of dense gas tracers in 
high-redshift galaxies. It would be the first interferometer capable of resolving the emission from the low- $J$ dense gas tracers at redshifts between $z \sim 1$ (roughly half the present-day age of the Universe), and to the end of the epoch of reionization at $z \sim 6$. In Section 5, we make predictions for the expected number of dense gas emitters that might be detected in future SKA1 and SKA2 spectral line surveys.

\section{4. $\mathrm{OH}$ and $\mathrm{H}_{2} \mathrm{O}$ megamaser emission}

Another potentially interesting probe of star-formation and the molecular ISM of high-redshift galaxies is redshifted emission from $\mathrm{OH}$ or $\mathrm{H}_{2} \mathrm{O}$ megamaser emission. Although it may be that the luminosity in the $\mathrm{OH}$ megamaser line is correlated with star-formation activity, this emission has not been detected in far-infrared luminous galaxies at high-redshift (e.g. Ivison 2006). Similarly, early searches for $\mathrm{H}_{2} \mathrm{O}$ megamasers at high-redshift (e.g. Wilner et al. 1999; Ivison 2006; Edmonds et al. 2009; Wagg \& Momjian 2009), resulted in only a pair of detections at $z=0.66$ (Barvainis $\&$ Antonucci 2005) and $z=2.64$ (Impellizzeri et al. 2008). It is therefore difficult to predict the expected number density of these line emitters with the increased sensitivity of the SKA. Murphy et al. (this edition) discuss the use of $\mathrm{H}_{2} \mathrm{O}$ megamaser line emission for studying AGN at highredshift.

\section{Predictions for SKA spectral line surveys}

For the purpose of predicting the expected detectability of molecular emission lines in future SKA observations, we first assume a $\Lambda$-dominated cosmological model with $\Omega_{\Lambda}=0.7, \Omega_{M}=0.3$, and $H_{0}=70 \mathrm{~km} \mathrm{~s}^{-1} \mathrm{Mpc}^{-1}$. The standard definition of line luminosity is given by Solomon, Downes \& Radford (1992), and we assume a linewidth, $F W H M=300 \mathrm{~km} \mathrm{~s}^{-1}$ throughout. For both SKA1 and SKA2, we consider two scenarios, one where targeted observations of known ultraluminous infrared galaxies (ULIRGs) are made $\left(L_{I R}=10^{12} \mathrm{~L}_{\odot}, S F R \sim 100 \mathrm{M}_{\odot} \mathrm{yr}^{-1}\right)$, and a second where pointed deep field observations are conducted, sensitive to less luminous galaxies. In the latter case, we adopt the models of da Cunha et al. (2013a), who make empirical predictions for the observed molecular line properties of star-forming galaxies in the observed Hubble Ultra Deep Field (UDF). In the original models, the expected CO line luminosity is predicted from the infrared luminosity, and here we extend this prediction to $\mathrm{HCN}, \mathrm{HCO}^{+}$, and $\mathrm{CS}$ which we convert from the $\mathrm{CO} J=1-0$ line luminosity by adopting the relationship to dense gas tracer line luminosity measured by Gao \& Solomon (2004).

For the purposes of SKA1 band 5 (4.6 to $13.8 \mathrm{GHz}$ ) predictions, we do not consider CO $\mathrm{J}=1-0$ line emission at $z>7.4$, due to the effects discussed in the previous section. ALMA observations of higher-J CO lines would be sensitive to the same star-forming molecular gas, while also benefitting from an increase in flux density that depends on $v^{2}$. For targeted observations of HCN $J=1-0$ or CS $J=1-0$ line emission in ULIRGs at $z \sim 5.4(\mathrm{HCN})$ or $z \sim 2.6$ (CS), the expected peak flux density in both cases would be $\sim 1.5 \mu \mathrm{Jy}$. A $5-\sigma$ detection of such a line would take $\sim 1000$ hours of integration time with the full SKA1, or $\sim 4000$ hours if SKA1 had 50\% less sensitivity. Now considering predictions for blind surveys, Figure 2 shows the expected number density of CS $J=1$ 0 line emitters as a function of flux density, where we also indicate the expected sensitity after 


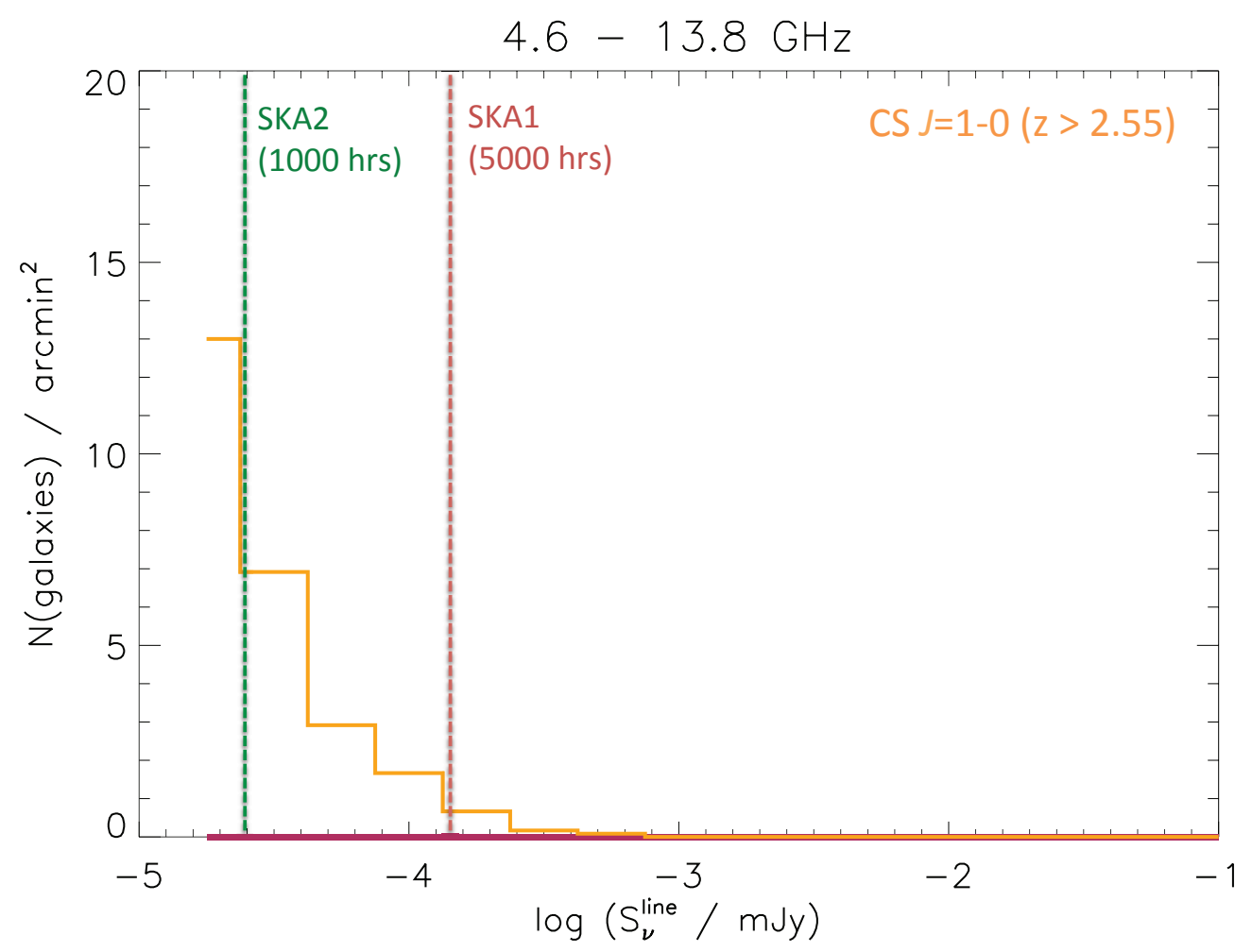

Figure 2: Predicted number density of CS $J=1-0$ line emitters (orange curve) as a function of peak flux density, assuming a $300 \mathrm{~km} \mathrm{~s}^{-1}$ linewidth. In a deep SKA1 survey (5000 hours integration time), one would expect to detect approximately one source per square arcminute over the field of view at $13.8 \mathrm{GHz}$. That number decreases to zero for SKA1 with half the sensitivity of the baseline design, but increases to 10 to 13 per square arcminute for an SKA2 survey (1000 hours integration time).

5000 hours with the full SKA1. In that case, one might expect to detect $\sim 1$ source per square arcminute over the field of view at the highest frequency end of the band.

SKA2 should enable a revolution in high-redshift molecular gas studies, not only because of the extended frequency coverage (up to $24 \mathrm{GHz}$ ), but also due to the increase from 190 to 2500 in the number of band 5 MID antennas. In assessing the potential for SKA2 studies, we only assume blank-field searches for molecular line emitting galaxies. We note that the expected rms in a $300 \mathrm{~km} \mathrm{~s}^{-1}$ channel after 1000 hours of on-source integration time is expected to be $\sim 24 \mathrm{nJy}$ at frequencies above $\sim 10 \mathrm{GHz}$. Figure 3 shows the predictions for two tunings, each covering $5 \mathrm{GHz}$ of bandwidth over the upper-end of SKA2-MID band 5. We only consider CO J=1-0 line emitters detected in the upper tuning $(z<5)$, when the emission from cold molecular gas is not expected to be dramatically effected by the CMB effects discussed previously. We note that at these redshifts metallicity evolution may have an impact on the detectability of $\mathrm{CO}$ line emission in galaxies with low metallicities. A deep integration covering the 19 to $24 \mathrm{GHz}$ frequency range is expected to detect more than $50 \mathrm{CO}$ line emitters per square arcminute. Such a survey would provide the first strong constraint on the evolution of the CO $J=1-0$ line luminosity function at $z>4$. 

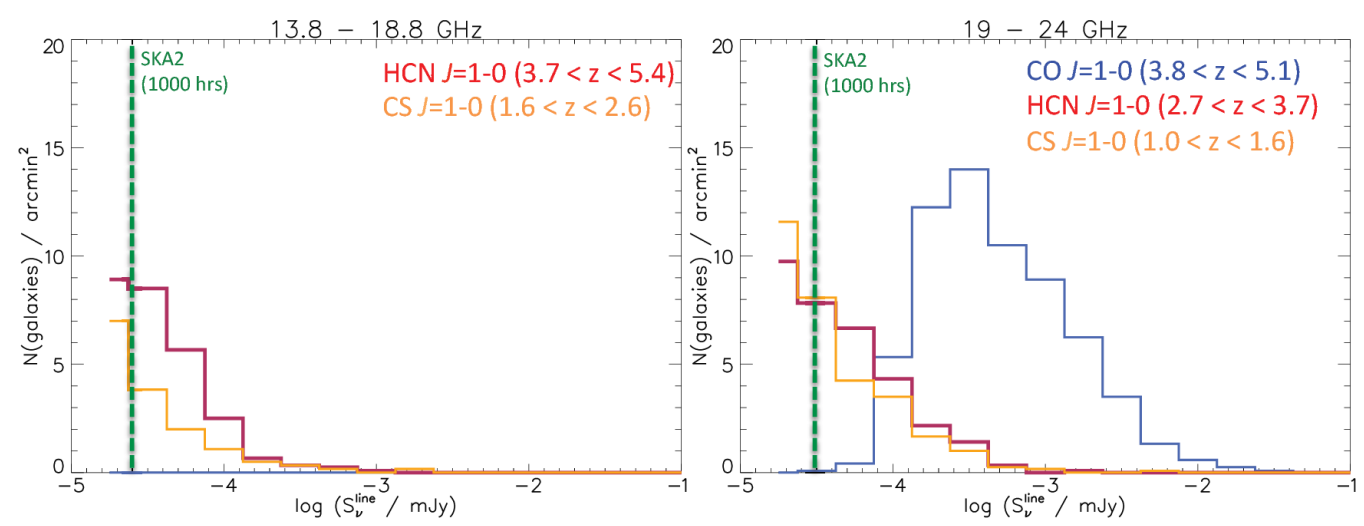

Figure 3: Predictions for the number density density of molecular line emitters as a function of peak line flux density (assuming a $300 \mathrm{~km} \mathrm{~s}^{-1}$ linewidth). The left panel shows the prediction for a $5 \mathrm{GHz}$ wide tuning between 13.8 and $18.8 \mathrm{GHz}$, while the right panel shows the same for a $5 \mathrm{GHz}$ wide tuning extending up to $24 \mathrm{GHz}$. CS $J=1-0$ line emitters are indicated by the orange curves, while the red curves show HCN $J=1-0$ (or $\mathrm{HCO}^{+} J=1-0$ ) and the blue curve in the right-hand panel indicates the expected number density of CO $J=1-0$ line emitters. Note that the decline in the number density of low luminosity CO line emitters is artificial, as it is based on the observed number density of low luminosity optically selected galaxies in the UDF.

\section{Summary}

The SKA has the potential to be a powerful facility for the detection and imaging of low- $J$ transitions of molecular line emission in high-redshift galaxies. During SKA1, it could be possible to detect faint CS $J=1-0$ line emission associated with $z>2.6$ luminous infrared galaxies $\left(L_{F I R}>\right.$ $10^{12} \mathrm{~L}_{\odot}$ ). At $z>5.4$, HCN $J=1-0$ could be observed in order to quantify the total dense molecular gas mass in galaxies. Our models predict that with the increased sensitivity and frequency coverage of SKA2 extending up to $\sim 24 \mathrm{GHz}$, we expect to detect significant numbers of $z>3.8 \mathrm{CO}$ line emitters in blank-field surveys. Such galaxies may be significant contributors to the total starformation rate density of the Universe at these early cosmic epochs.

\section{REFERENCES}

Aravena, M., Carilli, C., Daddi, E., et al. 2010, ApJ, 718, 177

Aravena, M., Hodge, J. A., Wagg, J., et al. 2014, MNRAS, 442, 558

Barvainis, R., \& Antonucci, R. 2005, ApJL, 628, L89

Bolatto, A. D., Wolfire, M., \& Leroy, A. K. 2013, ARA\&A, 51, 207

Carilli, C. L., \& Rawlings, S. 2004, New Astronomy Reviews, 48, 979

Carilli, C. L., Solomon, P., Vanden Bout, P., et al. 2005, ApJ, 618, 586

Carilli, C. L., Neri, R., Wang, R., et al. 2007, ApJL, 666, L9

Carilli, C. L., \& Walter, F. 2013, ARA\&A, 51, 105

Carilli, C. L., Riechers, D., Walter, F., et al. 2013, ApJ, 763, 120

Chary, R.-R., Stern, D., \& Eisenhardt, P. 2005, ApJL, 635, L5

da Cunha, E., Walter, F., Decarli, R., et al. 2013a, ApJ, 765, 9

da Cunha, E., Groves, B., Walter, F., et al. 2013b, ApJ, 766, 13 
Daddi, E., Dannerbauer, H., Elbaz, D., et al. 2008, ApJL, 673, L21

Daddi, E., Bournaud, F., Walter, F., et al. 2010, ApJ, 713, 686

Danielson, A. L. R., Swinbank, A. M., Smail, I., et al. 2011, MNRAS, 410, 1687

Dannerbauer, H., Daddi, E., Riechers, D. A., et al. 2009, ApJL, 698, L178

Decarli, R., Walter, F., Carilli, C., et al. 2014, ApJ, 782, 78

Di Francesco, J., Johnstone, D., Matthews, B. C., et al. 2013, arXiv:1310.1604

Downes, D., \& Solomon, P. M. 1998, ApJ, 507, 615

Edmonds, R., Wagg, J., Momjian, E., et al. 2009, AJ, 137, 3293

Evans, N. J., II 1999, ARA\&A, 37, 311

Gao, Y., \& Solomon, P. M. 2004, ApJS, 152, 63

Gao, Y., Carilli, C. L., Solomon, P. M., \& Vanden Bout, P. A. 2007, ApJL, 660, L93

García-Burillo, S., Graciá-Carpio, J., Guélin, M., et al. 2006, ApJL, 645, L17

Genzel, R., Tacconi, L. J., Combes, F., et al. 2012, ApJ, 746, 69

Impellizzeri, C. M. V., McKean, J. P., Castangia, P., et al. 2008, Nature, 456, 927

Ivison, R. J. 2006, MNRAS, 370, 495

Jarvis, M., Seymour, N., Afonso, J., et al., 2015, "The star-formation history of the Universe with the SKA", in Advancing Astrophysics with the SKA, PoS(AASKA14)068

Leroy, A. K., Bolatto, A., Bot, C., et al. 2009, ApJ, 702, 352

Maiolino, R., Cox, P., Caselli, P., et al. 2005, A\&A, 440, L51

Morganti, R., Sadler, E.M., and Curran, S.J., 2015, "Cool Outflows and HI absorbers with the SKA", in Advancing Astrophysics with the SKA, PoS(AASKA14)134

Murphy, E.J., Sargent, M.T., Beswick, R.J., et al., 2015, "The Astrophysics of Star Formation Across Cosmic Time at $>10 \mathrm{GHz}$ with the Square Kilometre Array", in Advancing Astrophysics with the SKA, PoS(AASKA14)085

Narayanan, D., et al. 2012, MNRAS, 421, 3127

Obreschkow, D., Heywood, I., Klöckner, H.-R., \& Rawlings, S. 2009, ApJ, 702, 1321

Obreschkow, D., Meyer, M., Popping, A., 2015, "The SKA as a Doorway to Angular Momentum", in Advancing Astrophysics with the SKA, PoS(AASKA14)138

Papadopoulos, P. P., Röttgering, H. J. A., van der Werf, P. P., et al. 2000, ApJ, 528, 626

Papadopoulos, P. P. 2007, ApJ, 656, 792

Prandoni, I., \& Seymour, N., 2015, "Revealing the Physics and Evolution of Galaxies and Galaxy Clusters with SKA Continuum Surveys", in Advancing Astrophysics with the SKA, PoS(AASKA14)067

Riechers, D. A., Walter, F., Carilli, C. L., et al. 2006, ApJL, 645, L13

Riechers, D. A., Walter, F., Carilli, C. L., \& Bertoldi, F. 2007, ApJL, 671, L13

Riechers, D. A., Bradford, C. M., Clements, D. L., et al. 2013, Nature, 496, 329

Riechers, D. A., Carilli, C. L., Capak, P. L., et al. 2014, arXiv:1404.7159

Santos, M., Bull, P., Alonso, D., et al., 2015, "Cosmology with a SKA HI intensity mapping survey" in Advancing Astrophysics with the SKA, PoS(AASKA14)019

Solomon, P., Vanden Bout, P., Carilli, C., \& Guelin, M. 2003, Nature, 426, 636

Staveley-Smith, L., et al., 2015, "The Hydrogen Universe with the SKA" in Advancing Astrophysics with the SKA, PoS(AASKA14) 167

Tacconi, L. J., Genzel, R., Neri, R., et al. 2010, Nature, 463, 781 
Tacconi, L. J., Neri, R., Genzel, R., et al. 2013, ApJ, 768, 74

Vanden Bout, P. A., Solomon, P. M., \& Maddalena, R. J. 2004, ApJL, 614, L97

Venemans, B. P., McMahon, R. G., Walter, F., et al. 2012, ApJL, 751, L25

Wagg, J., Wilner, D. J., Neri, R., Downes, D., \& Wiklind, T. 2005, ApJL, 634, L13

Wagg, J., Kanekar, N., \& Carilli, C. L. 2009a, ApJL, 697, L33

Wagg, J., \& Momjian, E. 2009b, AJ, 138, 895

Wagg, J., Carilli, C. L., Wilner, D. J., et al. 2010, A\&A, 519, L1

Wagg, J., Wiklind, T., Carilli, C. L., et al. 2012a, ApJL, 752, L30

Wagg, J., \& Kanekar, N. 2012b, ApJL, 751, L24

Walter, F., Bertoldi, F., Carilli, C., et al. 2003, Nature, 424, 406

Walter, F., Carilli, C., Bertoldi, F., et al. 2004, ApJL, 615, L17

Walter, F., Decarli, R., Sargent, M., et al. 2014, ApJ, 782, 79

Wang, R., Carilli, C. L., Neri, R., et al. 2010, ApJ, 714, 699

Wang, R., Wagg, J., Carilli, C. L., et al. 2013, ApJ, 773, 44

Weiß, A., Downes, D., Neri, R., et al. 2007, A\&A, 467, 955

Willott, C. J., Omont, A., \& Bergeron, J. 2013, ApJ, 770, 13

Wilner, D. J., Bourke, T. L., Ho, P. T. P., Killeen, N. E. B., \& Calabretta, M. 1999, AJ, 117, 1139

Wu, J., Evans, N. J., II, Gao, Y., et al. 2005, ApJL, 635, L173 